\title{
Neurobiología del trastorno de estrés postraumático
}

\author{
Jonathan A. Zegarra-Valdivia ${ }^{1}$ y Brenda N. Chino-Vilca ${ }^{1,2}$ \\ ${ }^{1}$ Universidad Nacional de San Agustín de Arequipa; ${ }^{2}$ Universidad Católica San Pablo, Arequipa, Perú
}

\section{Resumen}

A lo largo de la vida, las personas suelen estar expuestas a situaciones traumáticas, lo que en muchas ocasiones provoca la aparición del trastorno de estrés postraumático (TEPT), cuya prevalencia alcanza del 5 al $10 \%$ a nivel mundial. Sin embargo, pese a la gran cantidad de investigaciones que abordan el trastorno, la evidencia científica respecto a los procesos neurobiológicos aún no es concluyente. La presente revisión busca profundizar en el conocimiento de las características neurobiológicas más relevantes de los pacientes con TEPT. Se realiza una descripción de las áreas cerebrales involucradas y los efectos del TEPT en su funcionamiento, incluyendo un análisis del compromiso activo del eje hipotálamico-hipofisiario-adrenal, la hiperactivación y excesiva respuesta adrenérgica en el sistema nervioso autónomo, además de una breve descripción de los modelos animales más usados en las investigaciones. Se debe de tener en cuenta que la mayoría de los trastornos neuropsiquiátricos no comportan la alteración específica de una red neural, área o sistema, sino que en su conjunto presentan una alteración de distintos sistemas simultáneamente, que se solapan, y a su vez se hacen evidentes con endofenotipos neurocognitivos relativamente específicos, que apoyados en la investigación básica y la utilización con modelos animales proporcionaría los conocimientos de la alteración celular y molecular relacionada, aspectos significativos en la evaluación, tratamiento y seguimiento de los pacientes.

Palabras clave: Estrés. Trastorno de estrés postraumático. Neurobiología. Modelos animales. Revisión.

\section{Neurobiology review of post-traumatic stress disorder}

\begin{abstract}
Throughout life, people are often exposed to traumatic situations, causing the onset of post-traumatic stress disorder (PTSD), whose prevalence reaches 5-10\% worldwide. However, despite the large amount of research that addresses the disorder, the scientific evidence regarding neurobiological processes is not yet conclusive. The present review seeks to deep in the knowledge of the most relevant neurobiological characteristics of patients with PTSD. A description is made of the brain areas involved and the effects of PTSD on its functioning, including an analysis of the active commitment of the hypothalamic-pituitary-adrenal axis, hyperactivation and excessive adrenergic response in the autonomic nervous system, as well as a brief description of the animal models most used in research. It must be taken into account that the majority of neuropsychiatric disorders do not involve the specific alteration of a neural network, area or system, but as a whole they present an
\end{abstract}

\section{Correspondencia:}

Jonathan A. Zegarra-Valdivia

Universidad Nacional de San Agustín de

Arequipa

Venezuela Avenue, $\mathrm{s} / \mathrm{n}$

Fecha de recepción: 26-06-2018

Fecha de aceptación: 23-12-2018

C.P. 04000 , Cercado, Arequipa, Perú

E-mail: jzegarrav@unsa.edu.pe

DOI: 10.24875/RMN.M19000023
Disponible en internet: 20-03-2019

Rev Mex Neuroci. 2019;20(1):21-28

www.revmexneurociencia.com

1665-5044/@ 2019. Academia Mexicana de Neurología A.C. Publicado por Permanyer México. Este es un artículo Open Access bajo la licencia CC BY-NC-ND (http://creativecommons.org/licenses/by-nc-nd/4.0/). 
alteration of different systems simultaneously, which overlap and in turn become evident with relatively specific neurocognitive endophenotypes that, supported by basic research and use with animal models, would provide knowledge of the related cellular and molecular alteration, significant aspects in the evaluation, treatment, and follow-up of patients.

Key words: Animal models. Post-traumatic stress disorder. Neurobiology. Review. Stress.

\section{Introducción}

Vivimos una época inestable, caracterizada por ser altamente estresante y demandante. Percibimos distintos acontecimientos traumáticos de forma directa 0 indirecta (guerras, terrorismo, problemas económicos, robos/secuestros, accidentes de tráfico, violencia familiar o de pareja, feminicidios, e incluso catástrofes sobrenaturales y cambios climáticos, etc.), los cuales provocan secuelas y marcas emocionales que impactan en el desarrollo psicológico de las personas que los sufren. Entre los diversos trastornos que aparecen en la literatura, el más frecuentemente asociado al trauma es el trastorno de estrés postraumático (TEPT) ${ }^{1}$.

EI TEPT es un problema de salud pública, cuya prevalencia alcanza entre un 5 y un $10 \%^{2}$ y se eleva considerablemente en poblaciones en riesgo (personal militar/policial, madres maltratadas o niños en situación de peligro/abuso, etc.). Recientemente, la Organización Mundial de la Salud desarrolló un estudio con la participación de más de 24 países, en el cual determinó que el $70.4 \%$ de los evaluados sufrían experiencias traumáticas a lo largo de su vida, con un riesgo y persistencia de TEPT de 77.7 años por persona de cada 100 sujetos $^{3}$. Solo en Perú, la prevalencia del TEPT se sitúa entre el 14.6 y el $48.4 \%$, variando en función de los grupos evaluados ${ }^{4,5}$.

El trastorno tiene lugar después que el individuo se enfrenta con un estrés severo o evento traumático que implica la amenaza de muerte o daño significativo a uno mismo o a un ser querido. Es el único trastorno neuropsiquiátrico que puede ser relacionado directamente con un evento desencadenante al cual los pacientes responden con miedo y angustia intensos ${ }^{4}$. $\mathrm{Ha}$ sido especialmente estudiado en veteranos de guerra y supervivientes a accidentes automovilísticos, además de reportes en violencia, abandono infantil, maltrato y abuso sexual ${ }^{5,6}$. Sin embargo, no todos los individuos que experimentan un acontecimiento traumático desarrollan TEPT.

Se asocia con altos niveles de discapacidad social, laboral y física ${ }^{7}$ y ocasiona considerables costes económicos y alta utilización médica. Los pacientes con TEPT suelen tener peor salud y mayores limitaciones en la vida diaria en comparación con otros cuadros mentales ${ }^{8,9}$, y existe una mayor presencia de intentos de suicidio y enfermedades físicas ${ }^{10}$.

\section{Características clínicas del trastorno de estrés postraumático}

EI TEPT se caracteriza por la reexperimentación del trauma, comportamiento de evitación, alteraciones cognitivas y del estado del ánimo, así como por un incremento del arousal, que se manifiesta con irritación, hipervigilancia, sobresalto, alteraciones del sueño y pobre concentración ${ }^{7,9}$. Los pacientes muestran, además, sentimientos de ansiedad intensos y pueden revivir el evento traumático a través de los recuerdos intrusivos, flashbacks y pesadillas, por lo que evitan cualquier aspecto que les recuerda el trauma ${ }^{7}$. El trastorno posee una relación directa con el estatus metabólico, dado que suele presentar una alteración del eje hipotalámico-pituitario-adrenal (HPA) después del evento traumático ${ }^{11}$.

La comorbilidad en el TEPT es frecuente, así como la presencia de patología dual. Los trastornos mentales más prevalentes relacionados con el cuadro son la ansiedad, la depresión y el consumo de sustancias (alcohol, alucinógenos y psicotrópicos), los cuales alcanzan una comorbilidad de hasta un $50 \%$, incluso 10 años después del primer diagnóstico de TEPT ${ }^{12}$.

Las variaciones individuales en la presentación clínica y/o preclínica del TEPT dependen de factores genéticos ${ }^{13}$, neurobiológicos, neuropsicológicos, cambios epigenéticos y de las influencias ambientales ${ }^{14}$. Sin embargo, a pesar de la perspectiva clínica fenomenológica y el incremento de la investigación básica de las últimas décadas, los procesos neurobiológicos subyacentes no se comprenden del todo ${ }^{15,16}$, siendo el objetivo de la revisión profundizar en el conocimiento de las características neurobiológicas más relevantes de los pacientes con TEPT.

Se realizaron búsquedas en diferentes artículos originales, incluidos estudios experimentales, clínicos y de revisión, en las bases de datos MEDLINE, PubMed y Science Direct, publicados después del año 2000. Las palabras clave de búsqueda fueron: «trastorno de estrés postraumático", "estrés postraumático», «neurobiología», «neurociencia» y «modelos animales», siendo 
seleccionados únicamente aquellos estudios relevantes en el análisis de la neurobiología del TEPT.

\section{Desarrollo}

\section{Neurobiología del trastorno de estrés postraumático}

Como otros trastornos neuropsiquiátricos, el TEPT representa un estado de enfermedad multifacético ${ }^{17}$, en el cual distintas variables juegan un papel en su desarrollo y neurobiología. Diversas áreas como la corteza cerebral, el hipocampo, la amígdala o el hipotálamo se muestran alteradas en el TEPT ${ }^{18}$ y se interconectan para formar circuitos neuronales que median distintas funciones como la adaptación al estrés, la toma de decisiones y el miedo condicionado. A continuación revisaremos brevemente algunas de estas estructuras.

\section{Corteza PREFrontal}

La corteza prefrontal dorsal, la corteza prefrontal medial (mPFC) y el área cingulada anterior (CAA) están implicadas en una variedad de funciones, entre las que se señalan los procesos cognitivos de alto nivel, como la función ejecutiva, la cognición social, la regulación emocional y la regulación del eje HPA ${ }^{19,20}$. Los estudios de cambios neurofisiológicos del TEPT se han centrado principalmente en el mPFC, ya que jugaría un papel significativo al mediar la inhibición de la amígdala ${ }^{18}$ y la modulación de la respuesta neurohormonal al estrés ${ }^{21}$. Respecto a lo último, se ha reportado que las neuronas de la mPFC contraen sus dendritas y muestran una menor ramificación ${ }^{19,22}$, además de mostrar potenciales a largo plazo alterados ${ }^{23}$. Los hallazgos se correlacionan con un menor rendimiento en pruebas de memoria de trabajo y función ejecutiva ${ }^{24}$, y esto jugaría un papel clave en el aprendizaje de extinción de miedo ${ }^{25}$. Un posible mecanismo que media estos cambios involucra la exposición excesiva a glucocorticoides y glutamato ${ }^{19}$, explicado en otro apartado.

Los estudios de neuroimagen han identificado también alteraciones funcionales y estructurales asociadas al trastorno. Por ejemplo, en un estudio realizado con veteranos de guerra diagnosticados con TEPT, se observó un espesor cortical frontal reducido en ambos hemisferios ${ }^{26}$. Además, la evaluación de memoria de trabajo y activación de la corteza prefrontal, utilizando espectroscopia funcional de infrarrojo cercano, ha encontrado que los pacientes tenían una menor actividad frontal durante los procesos de codificación y recuperación ${ }^{27}$ ante las tareas de memoria. En las misma línea, los estudios con resonancia magnética funcional muestran peor conectividad frontoparietal, que se relaciona con una peor ejecución de tareas con componente atencional y de funciones ejecutivas en estos pacientes ${ }^{16}$.

\section{AmígdaLA}

La amígdala es una estructura vinculada con la emoción, con un papel crucial en la detección de amenazas y eventos emocionales ${ }^{28}$ relacionados con la adquisición y expresión del miedo condicionado. Esta estructura es capaz de perpetuar una respuesta de estrés mucho después de que un trauma ha terminado ${ }^{18,29}$, como se evidencia en un estudio realizado con ratas expuestas al olor de un depredador donde las células piramidales de la amígdala basolateral (BLA) mostraron una mayor longitud, número de dendritas y espinas $^{30}$. Se ha observado también un incremento del flujo sanguíneo en la amígdala relacionada con una mayor ansiedad y síntomas de TEPT durante la adquisición del miedo, así como una activación incrementada ante la reexposición a estímulos con contenido traumático ${ }^{21,31}$.

\section{HIPOCAMPO}

El hipocampo es una importante estructura relacionada con la memoria, el aprendizaje y la emoción. Es el principal objetivo neural para los glucocorticoides por las altas concentraciones de sus receptores ${ }^{32}$. Los glucocorticoides hacen sensible al hipocampo a elevados niveles de estrés, aceleran el envejecimiento y la atrofia del área, lo que sumado a la reducción de factores neurotróficos se relaciona con menor neurogénesis, perdida de la ramificación neuronal, perdida de espinas dendríticas y atrofia $21,30,32,33$.

Estudios con humanos muestran menores volúmenes de hipocampo en personas diagnosticadas con TEPT $^{21}$. Así, un estudio con imagen de resonancia magnética y con tomografía por emisión de positrones mostró que las mujeres que sufrieron abuso sexual en la infancia y desarrollaron TEPT tenían menor volumen del hipocampo $(-16 \%)$ y menor activación del área asociada a la memoria declarativa $(-19 \%$,) en comparación a mujeres que sufrieron abuso pero no desarrollaron el trastorno ${ }^{34}$.

La disfunción de las subregiones del hipocampo mediaría la sobregeneralización del miedo y los recuerdos intrusivos ${ }^{7,35}$; es el caso del hipocampo anterior, 
relacionando la disminución de la conectividad funcional con la corteza prefrontal y CAA con una mayor presencia de sintomatología de TEPT ${ }^{36}$.

\section{Hipotálamo e HIPÓFISIS}

El hipotálamo inicia distintos ejes de regulación metabólica y homeostática de gran importancia y complejidad, además de mediar la respuesta al estrés por medio del eje $\mathrm{HPA}^{37}$. Frente a un estresor, el núcleo paraventricular del hipotálamo sintetiza la hormona liberadora de corticotropina $(\mathrm{CRH})$. La CRH se libera hacia los vasos portales que acceden a la hipófisis anterior para unirse a su receptor e inducir la liberación de la hormona adrenocorticotropa (ACTH). El objetivo principal de la ACTH circulante es la corteza suprarrenal, donde estimula la síntesis de glucocorticoides al unirse a los receptores de mineralocorticoides en la corteza adrenal ${ }^{38}$.

El producto final de la actividad del eje HPA es el cortisol en humanos o la corticosterona en roedores ${ }^{39}$. El cortisol tiene una influencia directa en los circuitos cerebrales implicados en la homeostasis de la emoción, y a su vez controla la actividad del eje HPA. La respuesta al estrés ligada a la regulación del feedback negativo en el HPA estaría mediada por los receptores de glucocorticoides, mientras que los receptores de mineralocorticoides se encargarían de los estados basales del eje HPA (Fig. 1).

\section{EL EJE HIPOTALÁMICO-HIPOFISARIO-ADRENAL EN EL TEPT}

La actividad normal del eje HPA conduce a la liberación rítmica y episódica de glucocorticoides suprarrenales, proceso esencial para la homeostasis del cuerpo y la supervivencia durante el estrés, además de promover las respuestas de regulación de vías metabólicas, cardiovasculares, actividad inmunológica y neuroendocrina ${ }^{42}$. Los glucocorticoides cumplen la función de liberar sustratos de energía y así asegurar su disponibilidad en la oxidación mitocondrial para la respuesta de lucha o huida. Su sobreexposición altera el metabolismo afectando la acción de la insulina y generando su resistencia ${ }^{41}$.

Por otro lado, estudios iniciales mostraron discrepancias sobre los niveles de cortisol en el TEPT ${ }^{42}$. La evidencia señala niveles reducidos de cortisol durante el día y niveles elevados al anochecer ${ }^{43-45}$; es el caso del estudio desarrollado por Griffin, Resick y Yehuda ${ }^{46}$, donde los niveles de cortisol basales a las 9 a.m. mostraron una reducción significativa en mujeres con TEPT

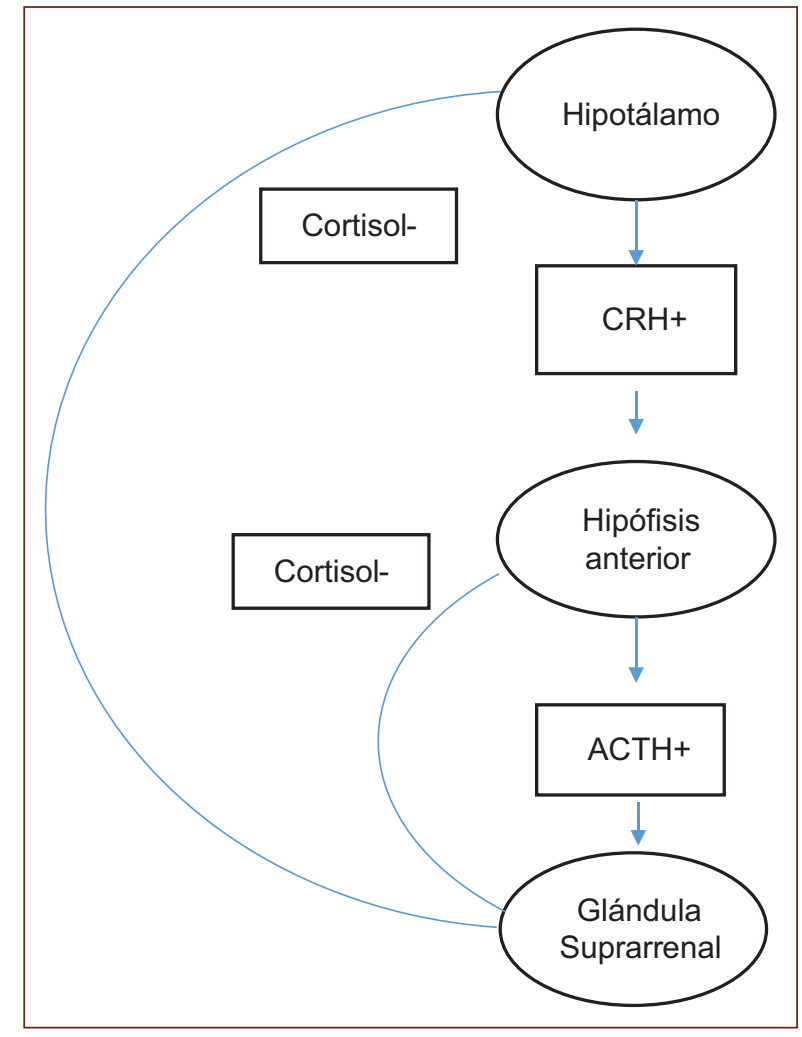

Figura 1. Eje hipotalámico-hipofisiario-adrenal en el TEPT.

que sufrieron violencia doméstica. Datos similares se han encontrado en sujetos que sufrieron abuso sexual en la infancia ${ }^{43}$, así como en veteranos de guerra diagnosticados ${ }^{47}$.

Lo referido sugiere que la alteración del eje HPA es una característica predominante de la fisiopatología del trastorno. Existen una amplia gama de condiciones que pueden alterar el eje HPA ${ }^{48}$ o sus receptores ${ }^{49}$, como polimorfismos genéticos, cambios en la expresión de genes por factores epigenéticos y condiciones ambientales, entre otras ${ }^{50}$.

\section{Test de supresión de dexametasona}

La dexametasona es un glucocorticoide sintético con acciones agonistas a los glucocorticoides, tiene una afinidad entre 20-30 veces mayor al cortisol y la corticosterona. Su mecanismo de acción incluye la supresión de la $\mathrm{CRH}$ y la $\mathrm{ACTH}{ }^{51}$ por medio de la unión a los receptores de glucocorticoides y provoca la activación del feedback negativo sobre la liberación de corticosterona. Además, muestra un limitado acceso al cerebro debido a la barrera hematoencefálica ${ }^{52}$. Su distribución y acción selectiva se da especialmente en la 
hipófisis, a través del sistema portal. En el TEPT, una supresión mayor del cortisol en respuesta a la dexametasona refleja un aumento de la respuesta de inhibición del eje HPA y sería reflejo del incremento en la sensibilidad de sus receptores ${ }^{48,53}$.

El test de supresión de dexametasona (TSD) ha sido ampliamente utilizado para evaluar el eje HPA en diversos trastornos psiquiátricos ${ }^{52}$. Es reconocido como medida para la detección de alteraciones funcionales en el sistema HPA por su fácil uso y su capacidad de detección de cambios fisiopatológicos. Ha sido usado en cuadros como la esquizofrenia, depresión y ansiedad, además de actuar como marcador biológico para el pronóstico de la respuesta del TEPT frente a la psicoterapia. Es el caso de Nijdman, et al., quienes reportaron un incremento de los niveles de supresión de cortisol, que se relacionan con mayor sintomatología y peor pronóstico, siendo las medidas de sensibilidad del eje HPA predictoras de la respuesta clínica positiva ${ }^{54}$.

También se han observado los efectos del TSD frente al miedo condicionado. Sus resultados sugieren que la supresión transitoria de la función HPA puede reducir el miedo exagerado en pacientes con TEPT ${ }^{55}$. Dosis bajas de dexametasona producen una activación selectiva de los receptores de glucocorticoides en la hipófisis mientras que no muestran una acción similar en el tejido cerebral ${ }^{52}$. Este uso concuerda con la idea de emplear glucocorticoides o inhibidores del receptor de glucocorticoides para potenciar la actividad del eje HPA como tratamiento en el TEPT ${ }^{53}$.

\section{Alteración del sistema nervioso autónomo en el TEPT}

Distintos estudios han abordado las alteraciones del sistema nervioso simpático (SNS) enfocando su atención en la hiperactivación y excesiva respuesta adrenérgica en el TEPT ${ }^{28}$. Las proyecciones noradrenérgicas que parten desde el locus cerúleo hacia la corteza prefrontal, la amígdala, el hipocampo y el hipotálamo, muestran una actividad incrementada ante un estresor ${ }^{56}$. Esta actividad moviliza recursos energéticos para la respuesta de lucha o huida. La noradrenalina, por ejemplo, aumenta la frecuencia cardíaca y el flujo de sangre a los músculos esqueléticos, y provoca la liberación de la glucosa. A su vez, desde el hipotálamo lateral, el sistema orexinérgico promueve la activación del locus cerúleos, propiciando cambios en la regulación de estado de sueño/vigilia, la emoción y la cognición en respuesta al miedo ${ }^{57}$.
En el TEPT, el SNS se caracteriza por una excesiva actividad noradrenérgica y esto se relaciona con mayor incidencia de enfermedad cardiovascular, mayor presencia de síntomas de TEPT y síndrome metabólico ${ }^{58}$. Además de asociarse a un respuesta de hipervigilancia, atención selectiva e hiperarousa ${ }^{59}$. La noradrenalina tiene un papel sustancial en la memoria a través de los glucocorticoides. Cuando la actividad noradrenérgica aumentada se asocia a la reducción de los glucocorticoides en la BLA, la respuesta al miedo condicionado se incrementa ${ }^{60}$. El eje HPA influencia también la actividad de la noradrenalina, mediante el incremento de $\mathrm{CRH}^{56}$. Esta respuesta implica el mantenimiento de los síntomas del TEPT como hiperactivación del SNS, refuerzo del miedo condicionado, pesadillas, flashbacks, etc.

\section{Sistemas de neurotransmisión implicados en el TEPT y el estrés}

Se ha observado que distintos sistemas de neurotransmisión se encuentran alterados en el TEPT. La presencia de un estresor impredecible durante el desarrollo aumenta los comportamientos de ansiedad en la edad adulta y se producen cambios en genes relacionados con la neurotransmisión de glutamato, ácido gamma aminobutírico (GABA), noradrenalina y serotonina, así como en sus respectivos receptores ${ }^{61}$. El condicionamiento y extinción al miedo se encuentran alterados en el TEPT y son mediados por el receptor de $\mathrm{N}$-metil-d-aspartato (NMDA), en la amígdala y la $\mathrm{mPFC}^{62}$. Las alteraciones de la plasticidad sináptica, retracción dendrítica y pérdida de espinas en la mPFC están asociadas con un exceso de activación de los receptores de NMDA por el aumento de los niveles de glutamato ${ }^{63}$; efectos similares se encontraron en el hipocampo de roedores sometidos a estrés agudo y severo ${ }^{64}$.

El glutamato en exceso en el cerebro provoca una amplia respuesta proinflamatoria y excitotoxicidad. En sujetos con TEPT se encontraron mayores niveles de glutamato en suero, menores ratios de glutamina/glutamato, asociados inversamente con la sintomatología del trastorno, y menores niveles de cortisol en saliva ${ }^{65}$. Sin embargo, el glutamato no se libera de forma aislada, algunos sistemas como el de la orexina lo utilizan de cotransmisor (actividad de orexina/glutamato, además de orexina/neurotensina, orexina/dinorfina, etc.), para generar una respuesta ante eventos de pánico, miedo o la regulación de la percepción del dolor y la analgesia ${ }^{66}$. 
Por otro lado, un desequilibrio entre el GABA y el glutamato provocarían una serie de cambios en la expresión de síntomas del TEPT. El incremento del glutamato puede promover la apoptosis en neuronas del hipocampo y la mPFC propiciando la patogénesis del TEPT ${ }^{64}$. Así mismo, los niveles disminuidos de GABA en suero ${ }^{67}$ podrían relacionarse con el estrés, la hiperexcitabilidad sináptica y la aparición de crisis convulsivas psicogénicas ${ }^{68,69}$.

\section{Modelos animales en el estudio del TEPT}

Para un estudio más detallado de las alteraciones neurobiológicas del TEPT se realizan distintos modelamientos del trastorno en animales, utilizando para tal fin la exposición a diversos estresores (inmovilización, nado forzado, footshock, inestabilidad del alojamiento, separación materna, etc.). No obstante, el abordaje de los mecanismos implicados en el desarrollo del TEPT requiere el estudio de modelos animales específicos que incorporen validez aparente y de constructo. Dichos modelos reflejan la sintomatología del TEPT de acuerdo con los hallazgos y características teóricas ya encontradas, así como las alteraciones neurobiológicas y neuroendocrinas, permitiendo una mayor comprensión de su fisiopatología ${ }^{28}$.

Dentro de los modelos animales específicos de TEPT destacan la exposición a depredador (ED), el estrés prolongado único (SPS, single prolonged stress) y el choque eléctrico con estresores adicionales ${ }^{28,70}$, los cuales mimetizan mejor las distintas características del TEPT. A continuación, describiremos cada uno de ellos:

- En el modelo de ED, los animales son expuestos a un depredador natural o a su olor en un medio en el que no pueda escapar ${ }^{28}$, reflejando el daño potencial al que se ven expuestos las personas que desarrollan TEPT $^{70}$. Los animales sometidos a los eventos simulan diversos criterios diagnósticos de TEPT, entre los que se observan una mayor ansiedad, una respuesta de sobresalto exagerada y déficits de memoria ${ }^{50}$, además de freezing y conducta de evitación ${ }^{17}$. Los síntomas persisten en el tiempo algunas semanas o incluso meses ${ }^{13}$. El modelo de ED refleja también las alteraciones en hipocampo, amígdala y mPFC, ${ }^{30}$ así como la desregulación del eje HPA, del sistema nervioso simpático ${ }^{17}$ y del sistema inmunitario, mostrando un incremento de la respuesta inflamatoria, con mayores niveles de TNF- $\alpha$, IL-6 y CD45 (un marcador de actividad microglial) en hipocampo e hipotálamo ${ }^{71}$.
- El SPS, contrariamente a su nombre, implica el uso de múltiples estresores, como la inmovilización durante dos horas, nado forzado durante 20 minutos y la exposición a éter hasta la pérdida de consciencia. Este modelo reproduce la alteración del eje HPA, con una disminución de los niveles de corticosterona, así como una sobreexpresión de los receptores de glucocorticoides $^{70}$. Se ha observado que representa también otros síntomas asociados al TEPT como la hiperexcitación, freezing y ansiedad ${ }^{19}$.

- El modelo de footshock con estresores adicionales utiliza un único episodio de descarga eléctrica asociado a un estímulo condicionado o recordatorios contextuales ${ }^{28}$. Dicho modelo muestra un incremento de la respuesta de sobresalto, hipersensibilidad noradrenérgica, disminución de la conducta social, inmovilidad, neofobia, etc. El uso de recordatorios contextuales incrementa la agresividad y la respuesta de sobresalto, además de disminuir la conducta exploratoria ${ }^{71}$.

Finalmente, los modelos animales del TEPT permiten realizar un estudio detallado de las alteraciones neurobiológicas del trastorno, neurológicas, del sistema inmunitario, endocrinas y metabólicas, donde estas últimas estarían ligadas a la alteración de factores neurotróficos, en relación a la actividad regulatoria del hipotálamo ${ }^{72}$.

\section{Conclusiones}

En esta breve revisión se han abordado los aspectos más relevantes ligados a la neurobiología del TEPT, un trastorno muy particular en la clínica debido a su distintiva característica de ser rastreable hasta un evento traumático en la vida del paciente. Este hecho es una herramienta única, debido a que se pueden estudiar los cambios en el cerebro con investigación en neurociencia básica y el modelamiento del trastorno en animales. Los mismos que, sumados a los datos aportados por pacientes, confirman una marcada alteración de la actividad prefrontal y cingulada (reducción de la corteza cerebral, disminución de dendritas e hipoactivación), de la amígdala (hiperactivación) y el hipocampo (especialmente atrofia).

Los cambios producto del TEPT se relacionan directamente con la actividad neurocognitiva de los pacientes, quienes muestran principalmente una alteración de las funciones ejecutivas (atención, memoria de trabajo, planificación, etc.) y de la regulación del estado del ánimo. Sin embargo, la alteración provocada por el trastorno no se limita a los procesos cognitivos y a sus 
estructuras subyacentes. Existen además zonas como el hipotálamo, relevante para el control de la homeostasis metabólica y endocrina del sujeto, que muestra cambios por medio de la actividad disfuncional del eje HPA, de los glucocorticoides (cortisol en sangre o saliva) y sus respectivos receptores (resistencia o disminución de receptores disponibles en áreas reguladoras como el hipocampo o el hipotálamo). Los cambios metabólicos alteran a su vez la capacidad de conservación y protección del cerebro, modificando el funcionamiento de distintos factores neurotróficos, muchos de ellos ligados al correcto funcionamiento del hipotálamo.

Se debe de tener en cuenta que la mayoría de los trastornos neuropsiquiátricos no comportan la alteración específica de una red neural, área o sistema, sino que en su conjunto presentan una alteración de distintos sistemas simultáneamente, que se solapan y a su vez se hacen evidentes con endofenotipos neurocognitivos relativamente específicos (funciones ejecutivas, toma de decisiones, teoría de la mente, coherencia central, cognición social, etc.). Sin embargo, su aproximación neurocognitiva aportará luces sobre los sistemas neurobiológicos alterados, y la investigación básica y la utilización con modelos animales proporcionaría los conocimientos de la alteración celular y molecular relacionada, aspectos significativos en la evaluación, tratamiento y seguimiento de estos pacientes.

Los actuales acontecimientos que sufre nuestra sociedad marcan la pauta de la salud mental de la población. Es por ello que debemos tener en cuenta el papel multifacético de los trastornos mentales (biopsicosocial) y cómo alteran las distintas actividades de la vida diaria de quienes sufren directa o indirectamente cada uno de estos problemas. A menudo, la población general presenta sintomatología subclínica que pasa desapercibida en atención primaria y que no recibe tratamiento oportuno durante años. Dicha situación genera un desborde de la capacidad de control mental y regulación emocional, soportando una problemática psicosocial relacionada (revictimización, violencia, desajuste socioemocional, suicidio, etc.). Por ello es importante la intervención multidisciplinaria, así como el desarrollo de políticas de salud pública en el ámbito socioemocional que lleguen a las poblaciones más vulnerables; un aspecto que desde la psiquiatría y la psicología sociocomunitaria, y de intervención en emergencias, es necesario promover.

\section{Declaración de conflictos de interés}

Los autores declaran que no existen conflictos de interés relevantes para este artículo de revisión.

\section{Fuentes de financiamiento}

No existió una fuente de financiamiento particular para este informe científico.

\section{Bibliografía}

1. Herrera-Lopez V, Cruzado L. Estrés postraumático y comorbilidad asociada en víctimas de la violencia política de una comunidad campesina de Huancavelica, Perú. 2013. Rev Neuropsiquiatr. 2014;77(3):144.

2. Gillespie C, Bradley B, Mercer K, Smith A, Conneely K, Gapen M, et al. Trauma exposure and stress-related disorders in inner city primary care patients. Gen Hosp Psychiatry. 2009;31(6):505-14.

3. Kessler RC, Aguilar-Gaxiola S, Alonso J, Benjet C, Bromet EJ, Cardoso 8, et al. Trauma and PTSD in the WHO World Mental Health Surveys. Eur J Psychotraumatol. 2017; 8(sup5):1353383.

4. Alani B, Maghsoudi N, Khatibi A, Noureddini M, Asefifar F, Shams J. Study of the variations in apoptotic factors in hippocampus of male rats with posttraumatic stress disorder. Adv Biomed Res. 2013;2(4):42.

5. Elzinga BM, Schmahl CG, Vermetten E, van Dyck R, Bremner JD. Higher cortisol levels following exposure to traumatic reminders in abuse-related PTSD. Neuropsychopharmacology. 2003;28(9):1656-65.

6. Aguirre P, Cova F, Domarchi MP, Garrido C, Mundaca I, Rincón P, et al. Estrés postraumático en mujeres víctimas de violencia doméstica. Rev Chil Neuropsiquiatr. 2010;48(2):114-22.

7. American Psychiatric Association. Diagnostic And Statistical Manual Of Mental Disorders, Fifth Edition; American Psychiatric Association Publishing; 2013.

8. Vermetten E, Lanius R a. Biological and clinical framework for posttraumatic stress disorder. Handb Clin Neurol. 2012;106:291-342.

9. Yehuda R, Hoge CW, McFarlane AC, Vermetten E, Lanius RA, Nievergelt CM, et al. Post-traumatic stress disorder. Nat Rev Dis Primers. 2015;1:15057.

10. Davidson JR, Hughes D, Blazer DG, George LK. Post-traumatic stress disorder in the community: an epidemiological study. Psychol Med. 1991; 21(3):713-21.

11. Daskalakis NP, Lehrner A, Yehuda R. Endocrine aspects of post-traumatic stress disorder and implications for diagnosis and treatment. Endocrinol Metab Clin North Am. 2013;42(3):503-13.

12. Arbanas G. Patients with combat-related and war-related posttraumatic stress disorder 10 years after diagnosis. Croat Med J. 2010;51(3):209-14.

13. Goswami S, Rodríguez-Sierra O, Cascardi M, Paré D. Animal models of post-traumatic stress disorder: Face validity. Front Neurosci. 2013;7:1-14.

14. Zannas AS, Provencal N, Binder EB. Epigenetics of posttraumatic stress disorder: Current evidence, challenges, and future directions. Biol Psychiatry. 2015;78(5):327-35.

15. Averill LA, Purohit P, Averill CL, Boesl MA, Krystal JH, Abdallah CG. Glutamate dysregulation and glutamatergic therapeutics for PTSD: Evidence from human studies. Neurosci Lett. 2017;649:147-55.

16. Liberzon I, Abelson JL. Context processing and the neurobiology of post-traumatic stress disorder. Neuron. 2016:92(1):14-30

17. Whitaker A, Gilpin N, Edwards S. Animal models of post-traumatic stress disorder and recent neurobiological insights. Behav Pharmacol. 2013; 18(9):1199-216.

18. Koenigs M, Grafmann J. Post-traumatic stress disorder: The role of medial prefrontal cortex and amygdala. Neuroscientist. 2009;15(5):540-8.

19. Moench KM, Wellman CL. Stress-induced alterations in prefrontal dendritic spines: Implications for post-traumatic stress disorder. Neurosci Lett. 2014:1-5

20. Zegarra-Valdivia J, Vilca BC. Mentalización y teoría de la mente. 2017; 80(3):189-99.

21. Bremner JD, Elzinga B, Schmahl C, Vermetten E. Structural and functional plasticity of the human brain in posttraumatic stress disorder. Prog Brain Res. 2008;167(07):171-86.

22. Radley JJ, Sisti HM, Hao J, Rocher AB, McCall T, Hof PR, et al. Chronic behavioral stress induces apical dendritic reorganization in pyramidal neurons of the medial prefrontal cortex. Neuroscience. 2004;125(1):1-6.

23. Maroun M. Medial prefrontal cortex: multiple roles in fear and extinction. Neuroscientist. 2013;19(4):370-83.

24. Hains AB, Vu MA, Maciejewski PK, van Dyck CH, Gottron M, Arnsten AF. Inhibition of protein kinase $\mathrm{C}$ signaling protects prefrontal cortex dendritic 
Rev Mex Neuroci. 2019;20

spines and cognition from the effects of chronic stress. Proc Natl Acad Sci U S A. 2009;106(42):17957-62.

25. Lisieski MJ, Eagle AL, Conti AC, Liberzon I, Perrine SA. Single-prolonged stress: A review of two decades of progress in a rodent model of post-traumatic stress disorder. Front Psychiatry. 2018;9:196.

26. Geuze E, Westenberg HGM, Heinecke A, de Kloet CS, Goebel R, Vermetten $\mathrm{E}$. Thinner prefrontal cortex in veterans with posttraumatic stress disorder. Neuroimage. 2008;41(3):675-81.

27. Tian F, Yennu A, Smith-Osborne A, Gonzalez-Lima F, North CS, Liu H. Prefrontal responses to digit span memory phases in patients with post-traumatic stress disorder (PTSD): A functional near infrared spectroscopy study. Neurolmage Clin. 2014;4:808-19.

28. Pitman RK, Rasmusson AM, Koenen KC, Shin LM, Orr SP, Gilbertson MW et al. Biological studies of post-traumatic stress disorder. Nat Rev Neurosci. 2012;13(11):769-87.

29. Arnsten AFT, Raskind MA, Taylor FB, Connor DF. The effects of stress exposure on prefrontal cortex: Translating basic research into successful treatments for post-traumatic stress disorder. Neurobiol Stress. 2015; 1:89-99.

30. Cohen H, Kozlovsky N, Matar MA, Zohar J, Kaplan Z. Distinctive hippocampal and amygdalar cytoarchitectural changes underlie specific patterns of behavioral disruption following stress exposure in an animal model of PTSD. Eur Neuropsychopharmacol. 2014;24(12):1925-44.

31. Liberzon I, Taylor SF, Amdur R, Jung TD, Chamberlain KR, Minoshima S, et al. Brain activation in PTSD in response to trauma-related stimuli. Biol Psychiatry. 1999;45(7):817-26.

32. Sapolsky RM, Uno H, Rebert CS, Finch CE. Hippocampal damage associated with prolonged glucocorticoid exposure in primates. J Neurosci. 1990;10(9):2897-902.

33. Mcewen BS, Bowles NP, Gray JD, Hill MN, Hunter RG, Karatsoreos IN and Nasca C. Mechanisms of stress in the brain. 2015;18(10):1353-63.

34. Bremner JD, Vythilingam M, Vermetten E, Southwick SM, McGlashan T, Nazeer A, et al. MRI and PET study of deficits in hippocampal structure and function inwomen with childhood sexual abuse and posttraumatic stress disorder. Am J Psychiatry. 2003;160(5):924-32.

35. Carrion VG, Wong SS. Can traumatic stress alter the brain? Understanding the implications of early trauma on brain development and learning J Adolesc Heal. 2012;51(2 SUPPL.):S23-8.

36. Malivoire BL, Girard TA, Patel R, Monson CM. Functional connectivity of hippocampal subregions in PTSD: relations with symptoms. BMC Psychiatry. 2018;18(1):129.

37. Mcewen BS. Physiology and neurobiology of stress and adaptation : Central role of the brain. Physiol Rev. 2007;87(3):873-904.

38. Smith S, Vale W. The role of the hypothalamic-pituitary-adrenal axis in neuroendocrine responses to stress. Dialogues Clin Neurosci. 2006; 8(May):383-95.

39. Hayes AS, Essex MJ, Oler JA, Davidson R J, Ruttle PL, Molloy EK, et al Developmental pathways to amygdala-prefrontal function and internalizing symptoms in adolescence. Nat Neurosci. 2012;15(12):1736-41.

40. Torres E, Aguilera G, Herman J, Fiedler J, Deak T, Cordeiro M. Nove aspects of glucocorticoid actions. J Neuroendocrinol. 2014;26(9):557-72.

41. Geer EB, Islam J, Buettner C. Mechanisms of glucocorticoid-induced insulin resistance: Focus on adipose tissue function and lipid metabolism. Endocrinol Metab Clin North Am. 2014;43(1):75-102.

42. Pitman RK, Lasko NB, Paulus LA, Yehuda R, Carson MA, Metzger LJ et al. Basal and suppressed salivary cortisol in female Vietnam nurse veterans with and without PTSD. Psychiatry Res. 2008;161(3):330-5.

43. Stein MB, Yehuda R, Koverola C, Hanna C. Enhanced dexmethasone suppression of plasma cortisol in adult women traumatized by childhood sexual abuse. Biol Psychiatry. 1997;42(96):680-6.

44. Morris MC, Rao U. Psychobiology of PTSD in the acute aftermath of trauma: Integrating research on coping, HPA function and sympathetic nervous system activity. Asian J Psychiatr. 2013;6(1):3-21.

45. Kallarackal AJ, Kvarta MD, Cai X, Cammarata E, Bailey AM, Thompson SM, et al. Chronic stress induces a selective decrease in AMPA receptor-mediated synaptic excitation at hippocampal temporoammonic-CA1 synapses. J Neurosci. 2013;33(40):15669-74.

46. Griffin MG, Resick PA, Yehuda R. Enhanced cortisol suppression following dexamethasone administration in domestic violence survivors. Am J Psychiatry. 2005;162(6):1192-9.

47. Yehuda R, Boisoneau D, Lowy MT, Giller EL. Dose-response changes in plasma cortisol and lymphocyte glucocorticoid receptors following dexamethasone administration in combat veterans with and without posttraumatic stress disorder. Arch Gen Psychiatry. 1995;52(7): 583-93.
48. Yehuda R. Status of glucocorticoid alterations in post-traumatic stress disorder. Ann N Y Acad Sci. 2009;1179:56-69.

49. Xiong F, Zhang L. Role of the hypothalamic-pituitary-adrenal axis in developmental programming of health and disease. Front Neuroendocrinol. 2013;34(1):27-46

50. Zovkic IB, Sweatt JD. Epigenetic mechanisms in learned fear: implications for PTSD. Neuropsychopharmacology. 2013;38(1):77-93.

51. Koerner KM. Establishing a protocol for dexamethasone suppression testing in mice. Honor Theses. 1997; Paper 205.

52. Cole MA, Kim PJ, Kalman BA, Spencer RL. Dexamethasone suppression of corticosteroid secretion: Evaluation of the site of action by receptor measures and functional studies. Psychoneuroendocrinology. 2000; 25(2):151-67.

53. Yehuda R, Golier J. Is there a rationale for cortisol-based treatments for PTSD? Expert Rev Neurother. 2009:9(8):1113-5.

54. Nijdam MJJ, van Amsterdam JGCGC, Gersons BPRPR, Olff M. Dexamethasone-suppressed cortisol awakening response predicts treatment outcome in posttraumatic stress disorder. J Affect Disord. 2015;184:205-8.

55. Jovanovic T, Phifer J, Sicking K, Weiss T, Norrholrn S, Bradley B, et al. Cortisol suppression by dexamethasone reduces exaggerated fear responses in posttraumatic stress disorder. Psychoneuroendocrinology. 2011;36(10):1540-52.

56. Strawn JR, Geracioti TD. Noradrenergic dysfunction and the psychopharmacology of posttraumatic stress disorder. Depress Anxiety. 2008; 25(3):260-71.

57. Flores Á, Saravia R, Maldonado R, Berrendero F. Orexins and fear: Implications for the treatment of anxiety disorders. Trends Neurosci. 2015;38(9):550-9.

58. Wilson CB, Ebenezer PJ, McLaughlin LD, Francis J. Predator exposure/ psychosocial stress animal model of post-traumatic stress disorder modulates neurotransmitters in the rat hippocampus and prefrontal cortex. PLoS One. 2014;9(2):e89104.

59. Lipov E, Kelzenberg B. Sympathetic system modulation to treat post-traumatic stress disorder (PTSD): A review of clinical evidence and neurobiology. J Affect Disord. 2012;142(1-3):1-5.

60. Tempesta D, Orsolini L, Fornaro M, Tomasetti C, Bartolomeis A, Sepede $G$, et al. Targeting the noradrenergic system in posttraumatic stress disorder: A systematic review and meta-analysis of prazosin trials. Curr Drug Targets. 2015;16(10):1094-106.

61. Sarro E, Sullivan R, Barr G. Unpredictable neonatal stress enhances adult anxiety and alters amygdala gene expression related to serotonin and GABA. Neuroscience. 2014;31(258):147-61.

62. Davis M. Translational research. NMDA Recept fear extinction Implic Cogn Behav Ther. 2011;(13):463-74.

63. Nishi D, Hashimoto K, Noguchi H, Hamazaki K, Hamazaki T, Matsuoka Y. Glutamatergic system abnormalities in posttraumatic stress disorder. Psychopharmacology (Berl). 2015;232(23):4261-8.

64. Gao J, Wang H, Liu Y, Li Y, Chen C, Liu L, et al. Glutamate and GABA imbalance promotes neuronal apoptosis in hippocampus after stress. Med Sci Monit. 2014;20:499-512.

65. Heidenreich JO, Seifert F, Bubner M, Colla M, Schubert F, Luborzewski A, et al. Glutamate as a spectroscopic marker of hippocampal structural plasticity is elevated in long-term euthymic bipolar patients on chronic lithium therapy and correlates inversely with diurnal cortisol. Mol Psychiatry. 2009;14(7):696-704.

66. Inutsuka A, Yamashita A, Chowdhury S, Nakai J, Ohkura M, Taguchi T, et al. The integrative role of orexin/hypocretin neurons in nociceptive perception and analgesic regulation. Sci Rep. 2016;6

67. Vaiva G, Thomas P, Ducrocq F, Fontaine M, Boss V, Devos P, et al. Low posttrauma GABA plasma levels as a predictive factor in the development of acute posttraumatic stress disorder. Biol Psychiatry. 2004;55(3):250-4.

68. Montel S, Cohn A, Donne C, Schwan R, Hingray C, El-Hage W, et al. Link between psychogenic nonepileptic seizures and complex PTSD: A pilot study. Eur J Trauma Dissociation. 2017;1(2):131-6.

69. Maguire J, Salpekar JA. Stress, seizures, and hypothalamic-pituitary-adrenal axis targets for the treatment of epilepsy. Epilepsy Behav. 2013, 26(3):352-62.

70. Liberzon I, Khan S, Young E, Cohen H, Matar M, Joseph Z. Animal models of post-traumatic stress disorder. En: Steckler T, Kalin N, Reul J, editores. Handbook Stress Brain. 2013;15:388-409.

71. Barnum CJ, Pace TWW, Hu F, Neigh GN, Tansey MG. Psychological stress in adolescent and adult mice increases neuroinflammation and attenuates the response to LPS challenge. J Neuroinflammation. 2012;9(1):9.

72. Zegarra-Valdivia JA. Insulin-like growth factor type 1 and its relation with neuropsychiatric disorders. Medwave. 2017;17(07):e7031. 